EPJ Web of Conferences 47, 01001 (2013)

DOI: $10.1051 /$ epjconf/20134701001

(C) Owned by the authors, published by EDP Sciences, 2013

\title{
WTS: A near-infrared transit survey
}

\author{
Brigitta Sipőcz ${ }^{1, a}$, Gábor Kovács ${ }^{2}$, Simon Hodgkin² ${ }^{2}$ David Pinfield ${ }^{1}$ \\ and WTS consortium ${ }^{3}$
}

\author{
${ }^{1}$ Centre for Astrophysics Research, University of Hertfordshire, College Lane, Hatfield \\ AL10 9AB, UK \\ ${ }^{2}$ Institute of Astronomy, University of Cambridge, Madingley Road, Cambridge CB3 OHA, UK \\ ${ }^{3}$ Various Institutes
}

\begin{abstract}
The WFCAM Transit Survey is a transiting planet survey running on the United Kingdom Infrared Telescope targeting M dwarf stars in the near infrared. The survey has been operating since 2007 and gathering photometric time series of about $15000 \mathrm{M}$ dwarf stars brighter than $\mathrm{J}=17 \mathrm{mag}$. We identified and followed-up planet candidates from the most complete field and found two hot Jupiters around non-M dwarf hosts (WTS-1b \& WTS-2b) but found no planets around the M dwarfs.
\end{abstract}

\section{OBSERVATIONS AND DATA QUALITY}

The WFCAM Transit Survey (WTS) is an ongoing campaign survey on the United Kingdom Infrared Telescope (UKIRT) operating in the near-infrared, in J-band since 2007. The WTS targets 4 superfields, two in the winter and two in the summer sky and each covering 1.6 square degrees in size. A field is made up of 8 pawprints with slightly overlapping regions at the edges. The WTS operates as a backup program of the large UKIRT surveys (such as the UKIDSS surveys and UHS). The WTS runs in queue mode using 1 or 0.5 hour long observing blocks resulting 4 or 2 epochs per block respectively. The exposure time is $90 \mathrm{~s}$. Table 1 summarizes main properties of the fields and the current number of epochs. We expect to complete ${ }^{1}$ 2-3 fields before the closure of UKIRT in 2013.

The main science goal to identify planets orbiting around $\mathrm{M}$ dwarfs or earlier stars. Other science topics includes but not limited to characterizing of low mass eclipsing binaries; studying $\mathrm{M}$ dwarf variability in the $\mathrm{J}$ band; identifying high proper motion sources e.g. cool white dwarfs, brown dwarfs, TNOs.

Figure 1 summarizes our light curve noise properties in the $19 \mathrm{hr}$ field. The saturation appears at $\mathrm{J}=13 \mathrm{mag}$. We reach 3-4 mmag precision at the bright end and at $\mathrm{J}=17 \mathrm{mag}$ where the sky noise dominates we have a precision of $2 \%$. Mainly because of follow-up possibilities, we limit our main science goal of finding planets to stellar sources brighter than $\mathrm{J}=17$ magnitude, but for other science topics we used fainter sources, too. For reference we show the signal depth for a few star-planet systems.

In Figure 2 we present a basic sensitivity diagram, showing the effect of the irregular observing pattern and number of epochs in the different fields. We used a simple simulation of a Neptune sized planet transiting around an M0 star. The simulation provides us an unbiased sample of different transit lengths and timings derived from random geometry of inclination (we allow grazing systems, too).

\footnotetext{
ae-mail: b.sipocz@herts.ac.uk

${ }^{1}$ A field is considered complete when it get 1000 or more observed epochs.

This is an Open Access article distributed under the terms of the Creative Commons Attribution License 2.0, which permits unrestricted use, distribution, and reproduction in any medium, provided the original work is properly cited.
} 
EPJ Web of Conferences

Table 1. WTS fields and number of epochs taken up to November 2012. Thenumber of stars is also listed (brighter than $\mathrm{J}=17 \mathrm{mag}$ ) altogetherwith the number of $\mathrm{M}$ dwarfs (more details in Section 2).

\begin{tabular}{ccccccc}
\hline field & $\begin{array}{c}\text { coordinates } \\
\text { RA, DEC }\end{array}$ & $\begin{array}{c}\text { galactic } \\
\text { l, b }\end{array}$ & $\begin{array}{c}\text { No. of } \\
\text { epochs }\end{array}$ & $\begin{array}{c}\text { objects } \\
(\mathrm{J}<17 \text { mag })\end{array}$ & $\begin{array}{c}\text { stellar } \\
\text { objects }\end{array}$ & $\begin{array}{c}\text { M } \\
\text { dwarfs }\end{array}$ \\
\hline 19 & $19.6+36.4$ & $70.0+07.8$ & 1383 & 69161 & 59270 & 6495 \\
17 & $17.2+03.7$ & $24.9+23.1$ & 970 & 17103 & 15343 & 2482 \\
07 & $07.1+12.9$ & $202.9+8.9$ & 785 & 24153 & 21224 & 3135 \\
03 & $03.6+39.2$ & $155.0-13.0$ & 627 & 17221 & 15159 & 3498 \\
\hline
\end{tabular}

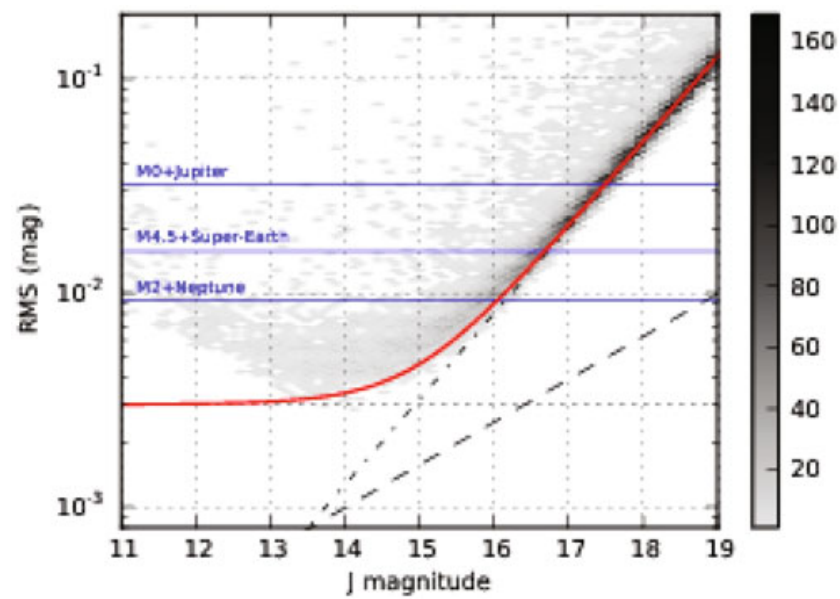

Figure 1. RMS diagram of the $19 \mathrm{hr}$ field, with expected signal depths. The red solid line is our noise model consisting of Poisson noise, sky noise and systematic noise.

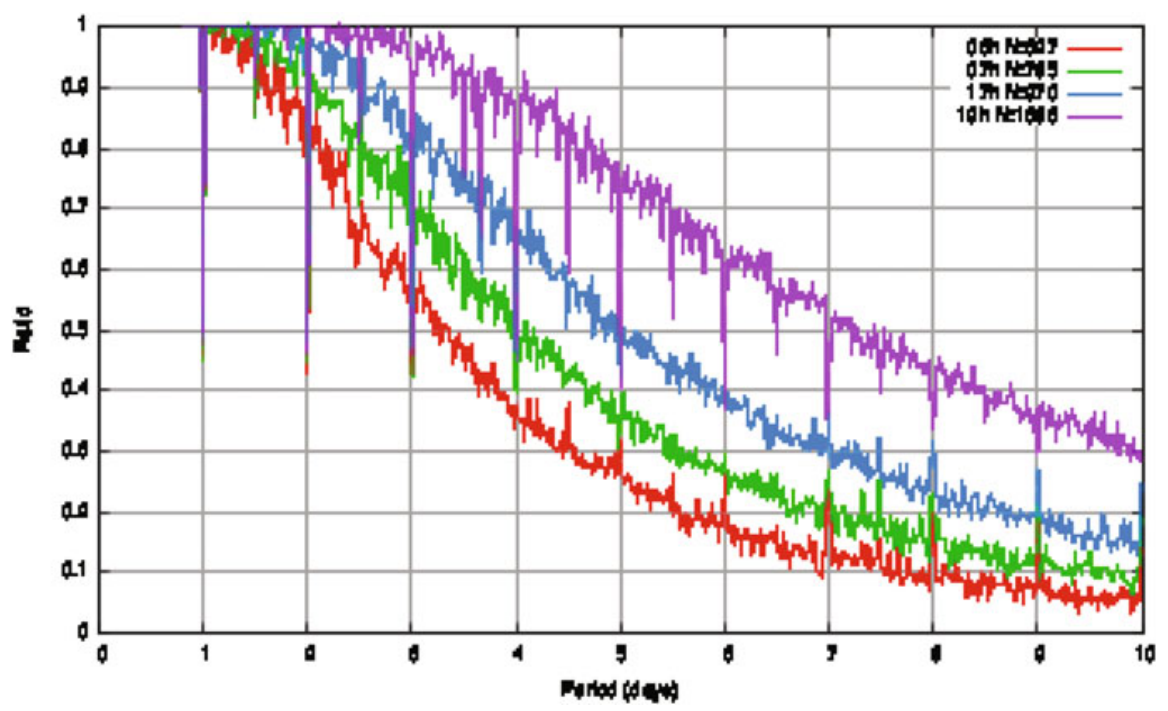

Figure 2. The effect of the observing data pattern and number of epochs on the sensitivity of WTS. A fraction of simulated transiting systems is shown here where there are at least 10 observed in-transit epochs. 

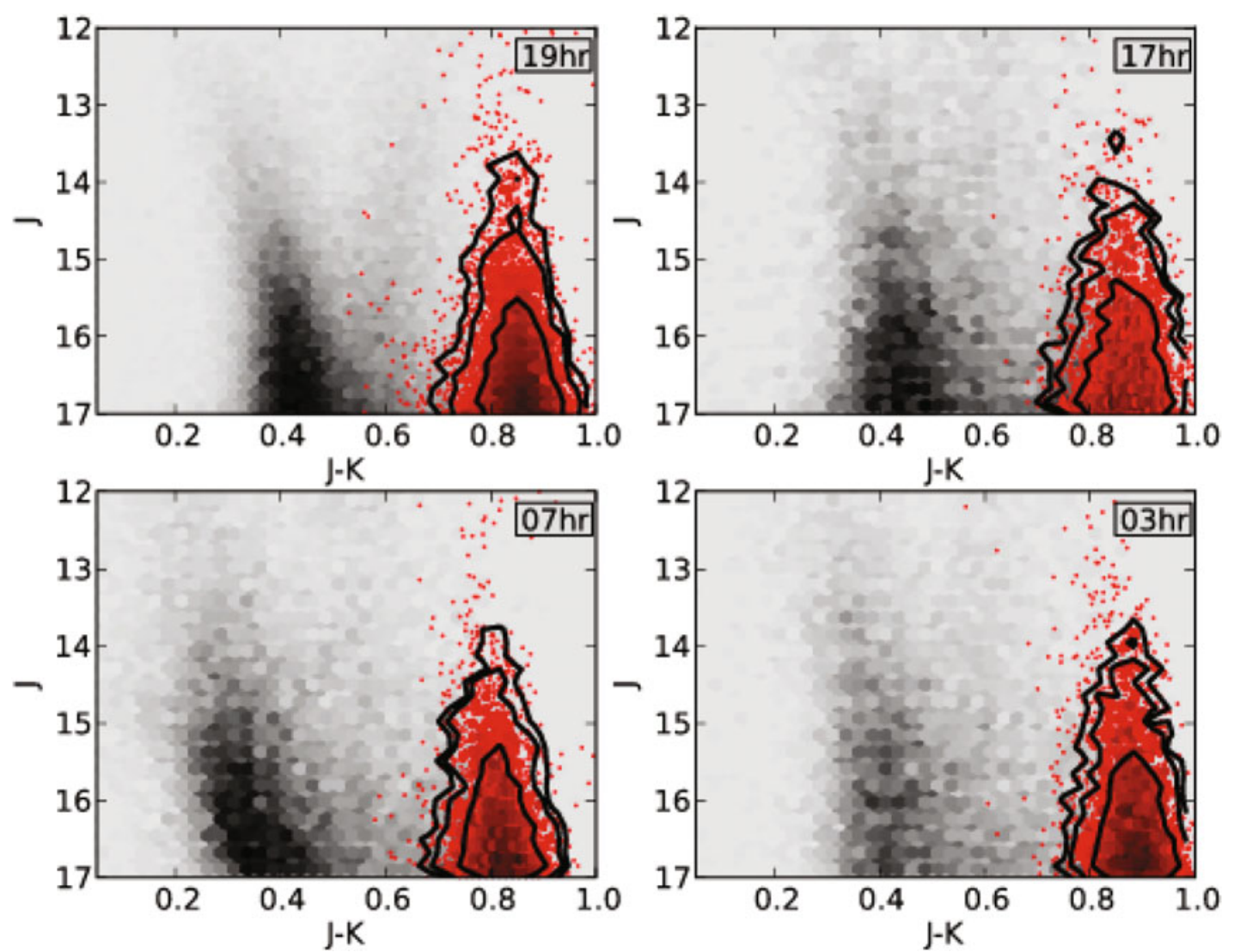

Figure 3. Colour-magnitude diagram for all the four WTS fields, red points are the selected M dwarf stars. Contours of $68 \%, 90 \%$ and $95 \%$ of the $\mathrm{M}$ dwarfs are shown for each fields.

We compared the simulated transit times with our real observational epochs and required to observe at least 10 in-transit data points for the recovery of a system. A more realistic estimation of planet recovery depends on the real system and noise properties. Our detailed sensitivity results are described in [1].

\section{M DWARF SAMPLE}

The WTS collects light curves of about 110000 stars brighter than $\mathrm{J}=17$ magnitude in the four fields, 15500 of them were identified as possible M dwarfs (Table 1.).

M dwarfs were selected using their estimated effective temperature. For three of the WTS fields SDSS photometry is available, and we used the SDSS ( $, r, \mathrm{r}, \mathrm{z})$ and the WFCAM (Z, Y, J, H, K) photometry through a spectral energy distribution (SED) fitting to obtain the temperature values. Objects brighter than $\mathrm{J}<13$ magnitude have unreliable classification because of saturation (in one or more of the 9 filters) and may have saturated J-band light curves. The total number of $\mathrm{M}$ dwarf stars with time series for all the four fields is about 15500 with $\mathrm{J}<17$ and Teff $<4000 \mathrm{~K}$. The majority of these objects are early M-dwarfs (M0-M4), and about 60\% of them are M0-M2 (Figure 3).

Compared to other surveys the WTS has a good balance in the sample size and time coverage. Other surveys are either monitoring smaller number of stars; e.g. the MEarth [2] monitors $2000 \mathrm{M}$ dwarfs individually in a broad $(\mathrm{i}+\mathrm{z})$-band while there are $1086 \mathrm{M}$ dwarfs identified in the Kepler Q2 [3]; or have much smaller number of timespan/epochs; the Palomar Transient Factory's (PTF, [4]) goal to 


\section{EPJ Web of Conferences}

observe 100,000 M dwarf with 300 epochs. PTF operates in the R band and covers similar magnitude range as the WTS.

\section{CANDIDATES}

We use the BLS algorithm to search for periodic transit signals in all the stellar light curves with magnitudes brighter than $\mathrm{J}=17 \mathrm{mag}$. For selecting and prioritizing candidates we use a heuristic scoring system which takes into account several light curve and BLS parameters as well as some derived parameters as consistency checks. The testbed for the system was a large set of simulated transits described in [5] and [1].

Follow-up observations are carried out for the candidates. Follow-ups include obtaining high cadence optical transit light curve, primarily with the INT and LT telescopes in La Palma. Spectroscopic follow-up includes medium resolution RV (with WHT/ISIS or CAHA/TWIN) and high resolution RV (HET/HDS or Gemini/GNIRS). In the $19 \mathrm{hr}$ field we ruled out all but two planet candidates, they are published and named WTS-1b [6] and WTS-2b [7]. We did not find any planets around M dwarfs. In addition to the planet candidates we identified about 70 low mass eclipsing binaries of which 14 were followed up spectroscopically to provide mass measurements.

\section{CONCLUSIONS}

The WTS is a unique infrared transit survey operated on the UKIRT telescope. By the time of termination in late 2013 we will have $\mathrm{J}$ band time series data spanning over 6 years. Our aim is to publish the multi-epoch data catalogues including photometry and astrometry. Our survey proved that ground based infrared surveys with poor sky irregular observing patterns are capable of finding exoplanets, however the follow-up of such deep surveys are very challenging.

\section{References}

[1] G. Kovács, S. Hodgkin, B. Sipőcz, D. Pinfield, et al, MNRAS submitted (2013)

[2] Z.K. Berta, J. Irwin, D. Charbonneau, C.J. Burke, E.E. Falco, AJ, 144, 145 (2012)

[3] A.W. Howard, G.W. Marcy, S.T. Bryson, J.M. Jenkins, J.F. Rowe, N.M. Batalha, W.J. Borucki, D.G. Koch, E.W. Dunham, T.N. Gautier, III et al., ApJSupp, 201, 15 (2012)

[4] N.M. Law, A.L. Kraus, R. Street, B.J. Fulton, L.A. Hillenbrand, A. Shporer, T. Lister, C. Baranec, J.S. Bloom, K. Bui et al., ApJ, 757, 133 (2012)

[5] B. Sipőcz et al., in preparation (2013)

[6] M. Cappetta, R.P. Saglia, J.L. Birkby, J. Koppenhoefer, D.J. Pinfield, S.T. Hodgkin, P. Cruz, G. Kovács, B. Sipőcz, D. Barrado et al., MNRAS, 427, 1877 (2012)

[7] J. Birkby, et al., MNRAS submitted (2013) 